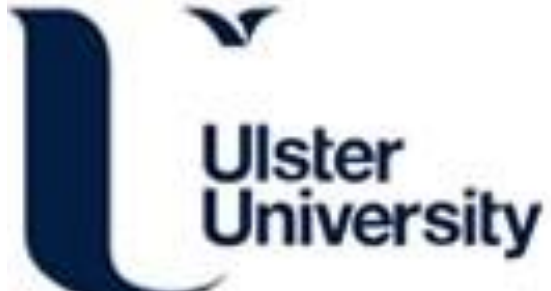

\section{Resource Allocation for Spectrum Sharing Cognitive Radio Networks}

Mohamed, E. B., Dobre, O. A., Ahmed, M. H., \& Baddour, K. E. (2013). Resource Allocation for Spectrum Sharing Cognitive Radio Networks. 2014 IEEE International Conference on Communications (ICC). https://doi.org/10.1109/ICC.2013.6654957

Link to publication record in Ulster University Research Portal

Published in:

2014 IEEE International Conference on Communications (ICC)

Publication Status:

Published (in print/issue): 07/11/2013

DOI:

10.1109/ICC.2013.6654957

\section{Document Version}

Author Accepted version

\section{General rights}

Copyright for the publications made accessible via Ulster University's Research Portal is retained by the author(s) and / or other copyright owners and it is a condition of accessing these publications that users recognise and abide by the legal requirements associated with these rights.

\section{Take down policy}

The Research Portal is Ulster University's institutional repository that provides access to Ulster's research outputs. Every effort has been made to ensure that content in the Research Portal does not infringe any person's rights, or applicable UK laws. If you discover content in the Research Portal that you believe breaches copyright or violates any law, please contact pure-support@ulster.ac.uk. 


\title{
Resource Allocation for Spectrum Sharing Cognitive Radio Networks
}

\author{
Ebrahim Bedeer, Octavia A. Dobre, Mohamed H. Ahmed, and Kareem E. Baddour ${ }^{\dagger}$ \\ Faculty of Engineering and Applied Science, Memorial University of Newfoundland, St. John's, NL, Canada \\ $\dagger$ Communications Research Centre, Ottawa, ON, Canada \\ Email: \{e.bedeer, odobre, mhahmed\}@mun.ca, kareem.baddour@crc.ca
}

\begin{abstract}
In this paper, we investigate the resource allocation problem (joint bit and power loading) of secondary users sharing the radio spectrum with primary users in cognitive radio networks. We consider the co-existence scenario where a secondary user is allowed to access the shared spectrum while guaranteeing tolerable interference to primary users. For such a scenario, we formulate and solve an optimization problem that jointly maximizes the secondary user throughput and minimizes its transmit power while satisfying target bit error rate per subcarrier and certain limits of co-channel and adjacent channel interferences to existing primary users. Simulation results are described that illustrate the performance of the proposed algorithm, and show its closeness to that of an exhaustive search for the equivalent discrete formulation.
\end{abstract}

Index Terms-Bit and power allocation, cognitive radio, dynamic spectrum sharing, joint optimization, OFDM systems, resource allocation.

\section{INTRODUCTION}

According to the recent measurements by the Federal Communications Commission (FCC), the current fixed spectrum allocation policies have resulted in severely underutilized spectrum bands [1] both temporally and/or spatially. This encouraged the FCC to allow unlicensed or secondary users (SUs) to share the licensed television spectrum when unused by licensed or primary users (PUs) [2]. Cognitive radio (CR) [3] generalizes this concept, by providing means to share the spectrum between SUs and PUs in order to alleviate the spectrum scarcity problem. One of the well-known approaches of spectrum sharing is to grant secondary users (SUs) access to the shared spectrum of primary users (PUs) under the condition that no harmful interference occurs to PUs [4].

Due to its flexibility, adaptivity in allocating vacant radio resources, and underlying sensing and spectrum shaping capabilities [5], orthogonal frequency division multiplexing (OFDM) has been recognized as a modulation of interest for cognitive radio (CR) systems. The performance of the OFDM-based CR systems can be significantly improved by dynamically loading different bits and/or power per each subcarrier according to the channel quality and the interference constraints to PUs [6][12]. The research literature has focused on the single objective of maximizing the OFDM SU capacity with constraints on the total transmit power and the interference introduced to adjacent PUs, while no attention has been given to guarantee a certain OFDM SU bit error rate (BER) [6]-[12]. In [6], Hasan et al. presented a novel solution to maximize the SU capacity while taking into account the availability of subcarriers, i.e. the activity of PUs in the licensed bands, and limit the interference leaked to PUs. Zhao and Kwak [7] maximized the capacity of the SU while keeping the interference to PUs below a certain threshold. A low-complexity iterative power loading algorithm and a suboptimal iterative bit loading algorithm were proposed to solve the modeled optimization problem.

Emerging wireless communication systems, including CR systems, operate under diverse conditions with different requirements. For example, minimizing the transmit power is prioritized when operating in interference-limited shared spectrum environments or in proximity to other frequencyadjacent users. On the other hand, maximizing the throughput is favored if sufficient guard bands exist to separate users. This motivates us to jointly maximize the throughput and minimize the transmit power by introducing a weighting factor that reflects the importance of the competing throughput and power objectives.

A novel close-to-optimal bit and power loading algorithm is proposed in this paper, which jointly maximizes the OFDM SU throughput and minimizes its transmit power ${ }^{1}$, while guaranteeing a SU target BER per subcarrier, total transmit power limit, and an acceptable interference power to adjacent PUs. Including the total subcarrier power in the objective function is especially desirable, as it minimizes the transmit power when the power constraint is inactive. Closed-form expressions are derived for the close-to-optimal bit and power distributions. Simulation results indicate that the proposed algorithm performance approaches that of an exhaustive search for the optimal discrete allocations, with significantly reduced computational effort.

The remainder of the paper is organized as follows. Section II introduces the system model and Section III presents the proposed joint bit and power loading algorithm. Simulation results are shown in Section IV, while conclusions are drawn in Section V.

Throughout this paper we use bold-faced lower case letters for vectors, e.g., $\mathbf{x}$, and light-faced letters for scalar quantities, e.g., $x .[.]^{T}$ denotes the transpose operation, $\nabla$ represents the gradient operator, and $\mathbb{E}[$.$] is the statistical expectation$ operator. $[x, y]^{-}$represents $\min (x, y)$ and $\overline{\bar{X}}$ is the cardinality of the set $\mathbb{X}$.

\footnotetext{
${ }^{1}$ In a non-CR environment, jointly maximizing the throughput and minimizing the transmit power show a significant performance improvement, in terms of the achieved throughput and transmit power, compared to other work in the literature that separately maximizes the throughput (while constraining the transmit power) or minimizes the transmit power (while constraining the throughput), respectively [13], [14].
} 


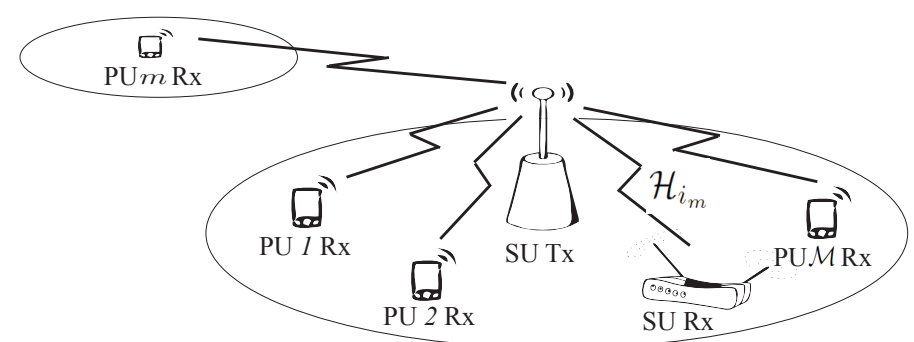

Fig. 1: Co-existence of an SU and $\mathcal{M}$ PUs in the spatial domain.

\section{SySTEM MODEL}

The available spectrum is assumed to be divided into $\mathcal{M}$ subchannels that are licensed to $\mathcal{M}$ PUs. A subchannel $m$, of bandwidth $B_{m}$, has $N_{m}$ subcarriers and $i_{m}$ denotes subcarrier $i$ in the subchannel $m, i_{m}=1, \ldots, N_{m}$. A PU does not occupy its licensed spectrum all the time and/or at all its coverage locations; hence, an SU may access such voids as long as no harmful interference occurs to adjacent PUs due to adjacent channel interference (ACI), or to other PUs operating in the same frequency band at distant locations due to co-channel interference $(\mathrm{CCI})$.

A typical CR system is shown in Fig. 1. An SU first obtains the surrounding PUs' information ${ }^{2}$, such as the PUs' positions and spectral band occupations. Then, it makes a decision on the possible transmission subchannels. We consider Fig. 1 where the SU has all the required information of the existing $\mathcal{M}$ PUs, and it decides to use the vacant PU $m$ subchannel, $m \in\{1, \ldots, \mathcal{M}\}$.

We assume that the instantaneous channel gains between the SU transmitter and receiver pairs are available through a delay- and error-free feedback channel. Further, we assume that the SU transmitter has perfect knowledge of the channel $\mathcal{H}_{s p}^{(\ell)}$ to the PU $\ell$ receiver as in [8]-[10]. This assumption is a reasonable approximation in the following scenarios: 1) in colocated co-existence scenarios, both the PU and SU receivers co-exist in the same user's device (for example, in future generation laptops, multiple radios will exist and one of these radios can be based on the $\mathrm{CR}$ technology) and, hence, the PU/SU receiver can estimate $\mathcal{H}_{s p}^{(\ell)}$ and report it to the SU transmitter [9], 2) the SU transmitter can estimate $\mathcal{H}_{s p}^{(\ell)}$ from the emitted signal from the PU $\ell$ receiver [9], and 3) the SU transmitter can estimate $\mathcal{H}_{s p}^{(\ell)}$ by eavesdropping the channel feedback between the PU $\ell$ transmitter and receiver pair [12].

In the following, we model both types of interference from the SU to the PUs (CCI and ACI). The interference, $\mathcal{J}_{i_{m}}$, from all the PUs to subcarrier $i_{m}$ of the SU is considered as in [6], [7], [9], [11], [15], which depends on the SU receiver windowing function and power spectral density (PSD) of the PU. $\mathcal{J}_{i_{m}}$ will not be presented here due to space limitations.

\section{A. Interference from the $S U$ to the PUs}

1) Co-channel interference (CCI): When the SU uses the $m$ subchannel, the total transmit power on this subchannel $\mathcal{P}_{T, m}$ should be less than a certain threshold $\mathcal{P}_{t h, m}$ at the location of the distant PU $m$ receiver. To further reflect the

\footnotetext{
${ }^{2}$ This is done by visiting a database administrated by a government or third party, or by optionally sensing the PUs' radio frequency [7].
}

SU transmitter's power amplifier limitations or/and to satisfy regulatory maximum power limits, the total SU transmit power should be limited to a certain threshold $\mathcal{P}_{t h}$. Hence, the condition on the total transmit power is formulated as

$\mathcal{P}_{T, m}=\sum_{i_{m}=1}^{N_{m}} \mathcal{P}_{i_{m}} \leq\left[\mathcal{P}_{t h},\left|\mathcal{H}_{s p}^{(m)}\right|^{-2} 10^{0.1 L\left(d_{m}\right)} \mathcal{P}_{t h, m}\right]^{-}$,

where $L\left(d_{m}\right)$ is the log-distance path loss in $\mathrm{dB}$ at distance $d_{m}$ [16] from the SU.

2) Adjacent channel interference (ACI): The ACI interference introduced to the PUs is caused by the sidelobe leakage of the SU subcarriers. Hence, this amount of interference depends on the power allocated to each SU subcarrier and the spectral distance between the SU subcarriers and the PUs. The total ACI from subcarrier $i_{m}$ of the SU to PU $\ell$ receiver can be formulated as [6], [7], [9], [11], [15]

$\mathcal{I}_{i_{m} \rightarrow \ell}=\mathcal{P}_{i_{m}} T_{s, m}\left|\mathcal{H}_{s p}^{(\ell)}\right|^{2} 10^{-0.1 L\left(d_{\ell}\right)} \int_{f_{i_{m}, \ell}-\frac{B_{\ell}}{2}}^{f_{i_{m}, \ell}+\frac{B_{\ell}}{2}} \operatorname{sinc}^{2}\left(T_{s, m} f\right) d f,(2)$ where $T_{s, m}$ is the duration of the OFDM symbol of the SU, $d_{\ell}$ is the distance from the $\mathrm{SU}$ and the PU $\ell$ receiver, $f_{i_{m}, \ell}$ is the spectral distance between the SU subcarrier $i_{m}$ and the $\mathrm{PU} \ell$ frequency band, $B_{\ell}$ is the bandwidth of the PU $\ell$, and $\operatorname{sinc}(x)=\frac{\sin (\pi x)}{\pi x}$. Consequently, the total ACI from the SU to the PU $\ell$ receiver should be kept below a certain threshold $\mathcal{P}_{\text {ACI }, \ell}$ as follow

$$
\sum_{i_{m}=1}^{N_{m}} \mathcal{I}_{i_{m} \rightarrow \ell}=\sum_{i_{m}=1}^{N_{m}} \mathcal{P}_{i_{m}} \varpi_{i_{m}}^{(\ell)} \leq \mathcal{P}_{\mathrm{ACI}, \ell}
$$

where $\varpi_{i_{m}}^{(\ell)}=T_{s, m}\left|\mathcal{H}_{s p}^{(\ell)}\right|^{2} 10^{-0.1 L\left(d_{\ell}\right)} \int_{f_{i_{m}, \ell}-\frac{B_{\ell}}{2}}^{f_{i_{m}, \ell}+\frac{B_{\ell}}{2}} \operatorname{sinc}^{2}\left(T_{s, m} f\right) d f$.

\section{Proposed Algorithm}

\section{A. Optimization Problem Formulation}

We propose a novel close-to-optimal algorithm that jointly maximizes the OFDM SU throughput and minimizes its transmit power, while satisfying a target BER per subcarrier ${ }^{3}$ and guaranteeing certain levels of $\mathrm{CCI} /$ total transmit power and ACI to adjacent PUs. The optimization problem is formulated as

$\underset{\mathcal{P}_{i_{m}}}{\operatorname{Minimize}} \mathcal{P}_{T, m}=\sum_{i_{m}=1}^{N_{m}} \mathcal{P}_{i_{m}}$ and $\underset{b_{i_{m}}}{\operatorname{Maximize}} b_{T, m}=\sum_{i_{m}=1}^{N_{m}} b_{i_{m}}$, subject to

$$
\begin{gathered}
\mathrm{BER}_{i_{m}} \leq \mathrm{BER}_{t h, i_{m}}, \quad i_{m}=1, \ldots, N_{m}, \\
\sum_{i_{m}=1}^{N_{m}} \mathcal{P}_{i_{m}} \leq\left[\mathcal{P}_{t h},\left|\mathcal{H}_{s p}^{(m)}\right|^{-2} 10^{0.1 L\left(d_{m}\right)} \mathcal{P}_{t h, m}\right]^{-} \\
i_{m}=1, \ldots, N_{m}, \quad \text { (4b) } \\
\sum_{i_{m}=1}^{N_{m}} \mathcal{P}_{i_{m}} \varpi_{i_{m}}^{(\ell)} \leq \mathcal{P}_{\mathrm{ACI}, \ell}, i_{m}=1, \ldots, N_{m}, \ell=1, \ldots, \mathcal{M},(4 \mathrm{c})
\end{gathered}
$$

where $b_{T, m}$ and $b_{i_{m}}$ are the throughput, and number of bits per subcarrier $i_{m}$, respectively, and $\mathrm{BER}_{i_{m}}$ and $\mathrm{BER}_{t h, i_{m}}$ are the BER per subcarrier $i_{m}$ and the threshold value of the BER per

\footnotetext{
${ }^{3}$ The constraint on the BER per subcarrier is a suitable formulation that results in similar BER characteristics compared to an average BER constraint, especially at high signal-to-noise ratios (SNR) [17]. Further, it enables obtaining closed-form expressions for the optimal bit and power solutions.
} 
subcarrier $i_{m}, i_{m}=1, \ldots, N_{m}$, respectively. An approximate expression for the BER per subcarrier $i_{m}$ in the case of $M$ ary QAM [18], while taking the interference from the PUs into account, is given by

$$
\mathrm{BER}_{i_{m}} \approx 0.2 \exp \left(-1.6 \frac{\mathcal{P}_{i_{m}}}{\left(2^{b_{i_{m}}}-1\right)} \frac{\left|\mathcal{H}_{i_{m}}\right|^{2}}{\left(\sigma_{n}^{2}+\mathcal{J}_{i_{m}}\right)}\right),
$$

where $\mathcal{H}_{i_{m}}$ is the channel gain of subcarrier $i_{m}$ between the SU transmitter and receiver pair and $\sigma_{n}^{2}$ is the variance of the additive white Gaussian noise (AWGN).

The multi-objective optimization problem can be rewritten as a linear combination of the multiple objective functions as follows

$$
\begin{aligned}
\underset{\mathcal{P}_{i_{m}}, b_{i_{m}}}{\text { Minimize }} & \mathcal{F}\left(\mathbf{p}_{m}, \mathbf{b}_{m}\right)=\alpha \sum_{i_{m}=1}^{N_{m}} \mathcal{P}_{i_{m}}-(1-\alpha) \sum_{i_{m}=1}^{N_{m}} b_{i_{m}}, \\
\text { subject to } & g_{\varrho}\left(\mathbf{p}_{m}, \mathbf{b}_{m}\right) \leq 0,
\end{aligned}
$$

where $\alpha(0<\alpha<1)$ is a constant whose value indicates the relative importance of one objective function relative to the other and determined according to the $\mathrm{CR}$ requirements/applications, i.e., minimum power versus maximum throughput, $\varrho=1, \ldots, N_{m}+2$ is the number of constraints, $\mathbf{p}_{m}=\left[\mathcal{P}_{1_{m}}, \ldots, \mathcal{P}_{N_{m}}\right]^{T}$ and $\mathbf{b}_{m}=\left[b_{1_{m}}, \ldots, b_{N_{m}}\right]^{T}$ are the $N_{m}$-dimensional power and bit distribution vectors, respectively, and

$$
\begin{aligned}
& g_{\varrho}\left(\mathbf{p}_{m}, \mathbf{b}_{m}\right)= \\
& \left\{\begin{array}{rr}
0.2 \sum_{i_{m}=1}^{N_{m}} b_{i_{m}} \exp \left(\frac{-1.6 \mathcal{C}_{i_{m}} \mathcal{P}_{i_{m}}}{2^{b_{m}}-1}\right)-\mathrm{BER}_{t h, i_{m}} \leq 0, \\
\varrho=i_{m}=1, \ldots, N_{m}, \\
\sum_{i_{m}=1}^{N_{m}} \mathcal{P}_{i_{m}}-\left[\mathcal{P}_{t h},\left|\mathcal{H}_{s p}^{(m)}\right|^{-2} 10^{0.1 L\left(d_{m}\right)} \mathcal{P}_{t h, m}\right]^{-} \leq 0,{ }^{(7)} \\
\\
\sum_{i_{m}=1}^{N_{m}} \mathcal{P}_{i_{m}} \varpi_{i_{m}}^{(\ell)}-\mathcal{P}_{\mathrm{ACI}, \ell} \leq 0, & \varrho=N_{m}+1,
\end{array}\right.
\end{aligned}
$$

where $\mathcal{C}_{i_{m}}=\frac{\left|\mathcal{H}_{i_{m}}\right|^{2}}{\sigma_{n}^{2}+\mathcal{J}_{i_{m}}}$ is the channel-to-noise-plusinterference ratio for subcarrier $i_{m}$.

\section{B. Optimization Problem Analysis and Solution}

The optimization problem in (6) can be solved by the method of Lagrange multipliers. Accordingly, the inequality constraints are transformed to equality constraints by adding non-negative slack variables, $\mathcal{Y}_{\varrho}^{2}, \varrho=1, \ldots, N_{m}+2$ [19]. Hence, the constraints are given as

$$
\mathcal{G}_{\varrho}\left(\mathbf{p}_{m}, \mathbf{b}_{m}, \mathbf{y}\right)=g_{\varrho}\left(\mathbf{p}_{m}, \mathbf{b}_{m}\right)+\mathcal{Y}_{\varrho}^{2}=0
$$

where $\mathbf{y}_{\varrho}=\left[\mathcal{Y}_{1}^{2}, \ldots, \mathcal{Y}_{N_{m}+2}^{2}\right]^{T}$ is the vector of slack variables, and the Lagrangian function $\mathcal{L}$ is expressed as

$$
\begin{aligned}
& \mathcal{L}\left(\mathbf{p}_{m}, \mathbf{b}_{m}, \mathbf{y}, \boldsymbol{\lambda}\right)=\mathcal{F}\left(\mathbf{p}_{m}, \mathbf{b}_{m}\right)+\sum_{\varrho=1}^{N_{m}+2} \lambda_{\varrho} \mathcal{G}_{\varrho}\left(\mathbf{p}_{m}, \mathbf{b}_{m}, \mathbf{y}\right), \\
& =\alpha \sum_{i_{m}=1}^{N_{m}} \mathcal{P}_{i_{m}}-(1-\alpha) \sum_{i_{m}=1}^{N_{m}} b_{i_{m}} \\
& \quad+\sum_{i_{m}=1}^{N_{m}} \lambda_{i_{m}}\left[0.2 \exp \left(\frac{-1.6 \mathcal{C}_{i_{m}} \mathcal{P}_{i_{m}}}{2^{b_{i_{m}}}-1}\right)-\mathrm{BER}_{t h, i_{m}}+\mathcal{Y}_{i_{m}}^{2}\right] \\
& \quad+\lambda_{N_{m}+1}\left[\sum_{i_{m}=1}^{N_{m}} \mathcal{P}_{i_{m}}-\left[\mathcal{P}_{t h},\left|\mathcal{H}_{s p}^{(m)}\right|^{-2} 10^{0.1 L\left(d_{m}\right)} \mathcal{P}_{t h, m}\right]^{-}\right.
\end{aligned}
$$

$$
+\lambda_{N_{m}+2}\left[\sum_{i_{m}=1}^{N_{m}} \mathcal{P}_{i_{m}} \varpi_{i_{m}}^{(\ell)}-\mathcal{P}_{\mathrm{ACI}, \ell}+\mathcal{Y}_{N_{m}+2}^{2}\right],
$$$$
\left.+\mathcal{Y}_{N_{m}+1}^{2}\right]
$$

where $\boldsymbol{\lambda}=\left[\lambda_{1}, \ldots, \lambda_{N_{m}+2}\right]^{T}$ is the vector of Lagrange multipliers associated with the $N_{m}+2$ constraints in (7). A stationary point is found when $\nabla \mathcal{L}\left(\mathbf{p}_{m}, \mathbf{b}_{m}, \mathbf{y}, \boldsymbol{\lambda}\right)=0$, which yields

$$
\begin{aligned}
& \frac{\partial \mathcal{L}}{\partial \mathcal{P}_{i_{m}}}=\alpha-\lambda_{i_{m}} \frac{(0.2)(1.6) \mathcal{C}_{i_{m}}}{2^{b_{i_{m}}}-1} \exp \left(\frac{-1.6 \mathcal{C}_{i_{m}} \mathcal{P}_{i_{m}}}{2^{b_{i_{m}}}-1}\right) \\
& +\lambda_{N_{m}+1}+\varpi_{i_{m}}^{(\ell)} \lambda_{N_{m}+2}=0 \text {, (10a) } \\
& \frac{\partial \mathcal{L}}{\partial b_{i_{m}}}=-(1-\alpha)+\lambda_{i_{m}} \frac{(0.2)(1.6)(\ln (2)) \mathcal{C}_{i_{m}} \mathcal{P}_{i_{m}} 2^{b_{i_{m}}}}{\left(2^{b_{i_{m}}}-1\right)^{2}} \\
& \times \exp \left(\frac{-1.6 \mathcal{C}_{i_{m}} \mathcal{P}_{i_{m}}}{2^{b_{i_{m}}}-1}\right)=0, \\
& \frac{\partial \mathcal{L}}{\partial \lambda_{i_{m}}}=0.2 \exp \left(\frac{-1.6 \mathcal{C}_{i_{m}} \mathcal{P}_{i_{m}}}{2^{b_{i_{m}}}-1}\right)-\mathrm{BER}_{t h, i_{m}}+\mathcal{Y}_{i_{m}}^{2} \\
& =0,(10 \mathrm{c}) \\
& \frac{\partial \mathcal{L}}{\partial \lambda_{N_{m}+1}}=\sum_{i_{m}=1}^{N_{m}} \mathcal{P}_{i_{m}}-\left[\mathcal{P}_{t h},\left|\mathcal{H}_{s p}^{(m)}\right|^{-2} 10^{0.1 L\left(d_{m}\right)} \mathcal{P}_{t h, m}\right]^{-} \\
& +\mathcal{Y}_{N_{m}+1}^{2}=0 \\
& \frac{\partial \mathcal{L}}{\partial \lambda_{N_{m}+2}}=\sum_{i_{m}=1}^{N_{m}} \mathcal{P}_{i_{m}} \varpi_{i_{m}}^{(\ell)}-\mathcal{P}_{\mathrm{ACI}, \ell}+\mathcal{Y}_{N_{m}+2}^{2}=0, \\
& \frac{\partial \mathcal{L}}{\partial \mathcal{Y}_{i, m}}=2 \lambda_{i_{m}} \mathcal{Y}_{i_{m}}=0 \\
& \frac{\partial \mathcal{L}}{\partial \mathcal{Y}_{N_{m}+1}}=2 \lambda_{N_{m}+1} \mathcal{Y}_{N_{m}+1}=0 \\
& \frac{\partial \mathcal{L}}{\partial \mathcal{Y}_{N_{m}+2}}=2 \lambda_{N_{m}+2} \mathcal{Y}_{N_{m}+2}=0
\end{aligned}
$$

It can be seen that (10) represents $4 N_{m}+4$ equations in the $4 N_{m}+4$ unknown components of the vectors $\mathbf{p}_{m}, \mathbf{b}_{m}, \mathbf{y}$, and $\lambda$. By solving (10), one obtains the solution $\mathbf{p}_{m}^{*}, \mathbf{b}_{m}^{*}$. Equation (10f) implies that either $\lambda_{i_{m}}=0$ or $\mathcal{Y}_{i_{m}}=0,(10 \mathrm{~g})$ implies that either $\lambda_{N_{m}+1}=0$ or $\mathcal{Y}_{N_{m}+1}=0$, and (10h) implies that either $\lambda_{N_{m}+2}=0$ or $\mathcal{Y}_{N_{m}+2}=0$. Hence, eight possible cases exist and we are going to investigate each case independently.

- Cases 1, 2, 3 , and 4: In (10), setting $\lambda_{i_{m}}=0$ and $\lambda_{N_{m}+1}=0$ (case 1)/ $/ \mathcal{Y}_{N_{m}+1}=0$ (case 2), or $\lambda_{N_{m}+2}=0$ (case 3) $/ \mathcal{Y}_{N_{m}+2}=0$ (case 4) result in an underdetermined system of $N_{m}+4$ equations in $3 N_{m}+2$ unknowns, and, hence, no unique solution can be reached.

- Case 5: Setting $\mathcal{Y}_{i_{m}}=\lambda_{N_{m}+1}=\lambda_{N_{m}+2}=0$ (i.e., inactive CCI/total transmit power and inactive ACI constraints), we can relate $\mathcal{P}_{i_{m}}$ and $b_{i_{m}}$ from (10a) and (10b) as follows

$$
\mathcal{P}_{i_{m}}=\frac{1-\alpha}{\alpha \ln (2)}\left(1-2^{-b_{i_{m}}}\right)
$$

with $\mathcal{P}_{i_{m}} \geq 0$ if and only if $b_{i_{m}} \geq 0$. By substituting (11) into (10c), one obtains the solution

$$
b_{i_{m}}^{*}=\frac{1}{\log (2)} \log \left[-\frac{1-\alpha}{\alpha \ln (2)} \frac{1.6 \mathcal{C}_{i_{m}}}{\ln \left(5 \mathrm{BER}_{t h, i_{m}}\right)}\right] .
$$


Consequently, from (11) one gets

$$
\mathcal{P}_{i_{m}}^{*}=\frac{1-\alpha}{\alpha \ln (2)}+\frac{\ln \left(5 \mathrm{BER}_{t h, i_{m}}\right)}{1.6 \mathcal{C}_{i_{m}}} .
$$

Since (5) is only valid for $M$-ary QAM, $b_{i_{m}}$ should be greater than 2. From (12), to have $b_{i_{m}} \geq 2$, the channel-to-noise ratio per subcarrier, $\mathcal{C}_{i_{m}}$, must satisfy the condition

$$
\mathcal{C}_{i_{m}} \geq \mathcal{C}_{t h, i_{m}}=-\frac{4}{1.6} \frac{\alpha \ln (2)}{1-\alpha} \ln \left(5 \mathrm{BER}_{t h, i_{m}}\right), i_{m}=1, \ldots, N_{m} \text {. }
$$

- Case 6: Setting $\mathcal{Y}_{i_{m}}=\mathcal{Y}_{N_{m}+1}=\lambda_{N_{m}+2}=0$ (i.e., active $\mathrm{CCI} /$ total transmit power and inactive $\mathrm{ACI}$ constraints), similar to case 5 , we obtain

$$
\begin{gathered}
\mathcal{P}_{i_{m}}=\frac{1-\alpha}{\ln (2)\left(\alpha+\lambda_{N_{m}+1}\right)}\left(1-2^{-b_{i_{m}}}\right), \\
b_{i_{m}}^{*}=\frac{1}{\log (2)} \log \left[-\frac{1-\alpha}{\ln (2)\left(\alpha+\lambda_{N_{m}+1}\right)} \frac{1.6 \mathcal{C}_{i_{m}}}{\ln \left(5 \mathrm{BER}_{t h, i_{m}}\right)}\right] \\
\mathcal{P}_{i_{m}}^{*}=\frac{1-\alpha}{\ln (2)\left(\alpha+\lambda_{N_{m}+1}\right)}+\frac{\ln \left(5 \mathrm{BER}_{t h, i_{m}}\right)}{1.6 \mathcal{C}_{i_{m}}}
\end{gathered}
$$

where $\lambda_{N_{m}+1}$ is calculated to satisfy the active $\mathrm{CCI} /$ total transmit power constraint in (10d). Hence, the value of $\lambda_{N_{m}+1}$ is found to be

$\lambda_{N_{m}+1}=\frac{\overline{\mathbb{N}}_{m}^{a} \frac{1-\alpha}{\ln 2}}{\left[\mathcal{P}_{t h},\left|\mathcal{H}_{s p}^{(m)}\right|^{-2} 10^{0.1 L\left(d_{m}\right) \mathcal{P}_{t h, m}}\right]^{-}-\sum_{i_{m} \in \mathbb{N}_{m}^{a}} \frac{\ln \left(5 \mathrm{BER}_{t h, i_{m}}\right)}{1.6 c_{i_{m}}}}$

where $\overline{\overline{\mathbb{N}}}_{m}^{a}$ is the cardinality of the set of active subcarriers $\mathbb{N}_{m}^{a}$.

- Case 7: Setting $\mathcal{Y}_{i_{m}}=\lambda_{N_{m}+1}=\mathcal{Y}_{N_{m}+2}=0$ (i.e., inactive $\mathrm{CCI} /$ total transmit power and active ACI constraints), similar to cases 5 and 6 , we obtain

$$
\begin{gathered}
\mathcal{P}_{i_{m}}=\frac{1-\alpha}{\ln (2)\left(\alpha+\varpi_{i_{m}}^{(\ell)} \lambda_{N_{m}+2}\right)}\left(1-2^{-b_{i_{m}}}\right), \\
b_{i_{m}}^{*}=\frac{1}{\log (2)} \log \left[-\frac{1-\alpha}{\ln (2)\left(\alpha+\varpi_{i_{m}}^{(\ell)} \lambda_{N_{m}+2}\right)} \frac{1.6 \mathcal{C}_{i_{m}}}{\ln \left(5 \mathrm{BER}_{t h, i_{m}}\right)}\right] .
\end{gathered}
$$

$$
\mathcal{P}_{i_{m}}^{*}=\frac{1-\alpha}{\ln (2)\left(\alpha+\varpi_{i_{m}}^{(\ell)} \lambda_{N_{m}+2}\right)}+\frac{\ln \left(5 \mathrm{BER}_{t h, i_{m}}\right)}{1.6 \mathcal{C}_{i_{m}}} .
$$

where $\lambda_{N_{m}+2}$ is calculated numerically using the Newton's method [20] to satisfy the active ACI constraint in (10e).

- Case 8: Setting $\mathcal{Y}_{i_{m}}=\mathcal{Y}_{N_{m}+1}=\mathcal{Y}_{N_{m}+2}=0$ (i.e., active $\mathrm{CCI} /$ total transmit power and active $\mathrm{ACI}$ constraints), similar to the previous cases, we obtain

$$
\begin{aligned}
\mathcal{P}_{i_{m}} & =\frac{1-\alpha}{\ln (2)\left(\alpha+\lambda_{N_{m}+1}+\varpi_{i_{m}}^{(\ell)} \lambda_{N_{m}+2}\right)}\left(1-2^{-b_{i_{m}}}\right), \\
b_{i_{m}}^{*} & =\frac{1}{\log (2)} \log \left[-\frac{1-\alpha}{\ln (2)\left(\alpha+\lambda_{N_{m}+1}+\varpi_{i_{m}}^{(\ell)} \lambda_{N_{m}+2}\right)}\right. \\
& \left.\frac{1.6 \mathcal{C}_{i_{m}}}{\ln \left(5 \mathrm{BER}_{t h, i_{m}}\right)}\right], \\
\mathcal{P}_{i_{m}}^{*}= & \frac{1-\alpha}{\ln (2)\left(\alpha+\lambda_{N_{m}+1}+\varpi_{i_{m}}^{(\ell)} \lambda_{N_{m}+2}\right)}+\frac{\ln \left(5 \mathrm{BER}_{t h, i_{m}}\right)}{1.6 \mathcal{C}_{i_{m}}},
\end{aligned}
$$

where $\lambda_{N_{m}+1}$ and $\lambda_{N_{m}+2}$ are calculated numerically using the Newton's method to satisfy the active CCI/total transmit power and ACI constraints in (10d) and (10e), respectively.

The obtained solution $\left(\mathbf{p}_{m}^{*}, \mathbf{b}_{m}^{*}\right)$ represents a minimum of $\mathcal{F}\left(\mathbf{p}_{m}, \mathbf{b}_{m}\right)$ as the Karush-Kuhn-Tucker (KKT) conditions
[19] are satisfied, as shown in Appendix $\mathrm{A}^{4}$.

\section{Proposed Joint Bit and Power Loading Algorithm}

The proposed algorithm can be formally stated as follows.

\section{Proposed Algorithm}

1: INPUT The AWGN variance $\left(\sigma_{n}^{2}\right)$, channel gain per subcarrier $i_{m}\left(\mathcal{H}_{i_{m}}\right)$, target BER per subcarrier $i_{m}\left(\mathrm{BER}_{t h, i_{m}}\right)$, initial weighting parameter $\alpha, \mathcal{P}_{t h}, \mathcal{P}_{t h, m}, \mathcal{P}_{\mathrm{ACI}, \ell}$, and PUs information.

2: for $i_{m}=1, \ldots, N_{m}$ do

3: $\quad$ if $\mathcal{C}_{i_{m}} \geq \mathcal{C}_{t h, i_{m}}=-\frac{4}{1.6} \frac{\alpha \ln (2)}{1-\alpha} \ln \left(5 \mathrm{BER}_{t h, i_{m}}\right)$ then

4: $\quad-\quad-b_{i_{m}}^{*}$ and $\mathcal{P}_{i_{m}}^{*}$ are given by (12) and (13), respectively.

$$
\text { 5: } \quad \text { else }
$$

6: $\quad$ Null the corresponding subcarrier $i_{m}$.

7: $\quad$ end if

8: end for

9: if $\sum_{i_{m}=1}^{N_{m}} \mathcal{P}_{i_{m}} \geq\left[\mathcal{P}_{t h},\left|\mathcal{H}_{s p}^{(m)}\right|^{2} 10^{0.1 L\left(d_{m}\right)} \mathcal{P}_{t h, m}\right]^{-}$and

$\sum_{i_{m}=1}^{N_{m}} \mathcal{P}_{i_{m}} \varpi_{i_{m}}^{(\ell)} \leq \mathcal{P}_{\mathrm{ACI}, \ell}$ then

10: $\quad-\quad-b_{i_{m}}^{*}$ and $\mathcal{P}_{i_{m}^{*}}^{*}$ are given by (16) and (17), respectively.

11: $\quad-\lambda_{N_{m}+1}$ is given by (18) and $\lambda_{N_{m}+2}=0$.

12: else if $\sum_{i_{m}=1}^{N_{m}} \mathcal{P}_{i_{m}} \leq\left[\mathcal{P}_{t h},\left|\mathcal{H}_{s p}^{(m)}\right|^{2} 10^{0.1 L\left(d_{m}\right)} \mathcal{P}_{t h, m}\right]^{-}$and $\sum_{i_{m}=1}^{N_{m}} \mathcal{P}_{i_{m}} \varpi_{i_{m}}^{(\ell)} \geq \mathcal{P}_{\mathrm{ACI}, \ell}$ then

13: $\quad-\quad-b_{i_{m}}^{*}$ and $\mathcal{P}_{i_{m}}^{*}$ are given by (20) and (21), respectively.

14: $\quad-\lambda_{N_{m}+1} \stackrel{0}{=} 0$ and $\lambda_{N_{m}+2}$ are calculated to satisfy $\sum_{i_{m}=1}^{N_{m}} \mathcal{P}_{i_{m}} \varpi_{i_{m}}^{(\ell)}=\mathcal{P}_{\mathrm{ACI}, \ell}$

15: else if $\quad \sum_{i_{m}=1}^{N_{m}} \mathcal{P}_{i_{m}} \geq\left[\mathcal{P}_{t h},\left|\mathcal{H}_{s p}^{(m)}\right|^{2} 10^{0.1 L\left(d_{m}\right)} \mathcal{P}_{t h, m}\right]^{-}$and $\sum_{i_{m}=1}^{N_{m}} \mathcal{P}_{i_{m}} \varpi_{i_{m}}^{(\ell)} \geq \mathcal{P}_{\mathrm{ACI}, \ell}$

16: $\quad-\quad-b_{i_{m}}^{*}$ and $\mathcal{P}_{i_{m}}^{*}$ are given by (23) and (24), respectively.

17: $\quad-\quad \lambda_{N_{m}+1}$ and $\lambda_{N_{m}+2}$ are calculated to satisfy $\sum_{i_{m}=1}^{N_{m}} \mathcal{P}_{i_{m}}=\left[\mathcal{P}_{t h},\left|\mathcal{H}_{s p}^{(m)}\right|^{2} 10^{0.1 L\left(d_{m}\right)} \mathcal{P}_{t h, m}\right]^{-}$and $\sum_{i_{m}=1}^{N_{m}=1} \mathcal{P}_{i_{m}} \varpi_{i_{m}}^{(\ell)}=\mathcal{P}_{\mathrm{ACI}, \ell}$, respectively.

\section{8: end if}

19: $-b_{i_{m}, f i n a l}^{*} \leftarrow$ Round $b_{i_{m}}^{*}$ to the nearest integer.

20: $-\mathcal{P}_{i_{m}, \text { final }}^{*} \leftarrow$ Recalculate $\mathcal{P}_{i_{m}}^{*}$ according to (5).

21: - If the conditions on the $\mathrm{CCI} /$ total transmit power and the $\mathrm{ACI}$ are violated due to rounding, decrement the number of bits on the subcarrier that has the largest $\Delta \mathcal{P}_{i_{m}}\left(b_{i_{m}}\right)=\mathcal{P}_{i_{m}}\left(b_{i_{m}}\right)-$ $\mathcal{P}_{i_{m}}\left(b_{i_{m}}-1\right)$ until satisfied.

22: OUTPUT $b_{i_{m}, \text { final }}^{*}$ and $\mathcal{P}_{i_{m}, \text { final }}^{*}, i_{m}=1, \ldots, N_{m}$.

\section{Numerical Results}

In this section, we present illustrative numerical results for the proposed allocation algorithm. Without loss of generality, we consider $\left[\mathcal{P}_{t h},\left|\mathcal{H}_{s p}^{(m)}\right|^{-2} 10^{0.1 L\left(d_{m}\right)} \mathcal{P}_{t h, m}\right]^{-}=\mathcal{P}_{t h}$, we assume that the OFDM SU coexists with one adjacent PU and one co-channel PU, and both SU and PUs have an equal bandwidth of $1.25 \mathrm{MHz}$. The OFDM SU transmission parameters are as follows: number of subcarriers $N_{m}=128$, symbol duration $T_{s, m}=102.4 \mu \mathrm{sec}$, and subcarrier spacing $\Delta f_{m}=9.7656 \mathrm{kHz}$. The propagation log-distance path loss parameters are as follows: exponent $\beta=4$, wavelength $人=\frac{3 \times 10^{8}}{900 \times 10^{6}}=0.33$ meters, distance to PU $\ell d_{\ell}=1 \mathrm{~km}$,

${ }^{4}$ Since the optimization problem in (6) is not convex, the obtained solution is not guaranteed to be a global optimum. In the next section, we compare the local optimum results to the global optimum results obtained through an exhaustive search to 1) characterize the gap to the global optimum solution and 2) characterize the gap to the equivalent discrete optimization problem (i.e., with integer constraints on $b_{i_{m}}$ ). 


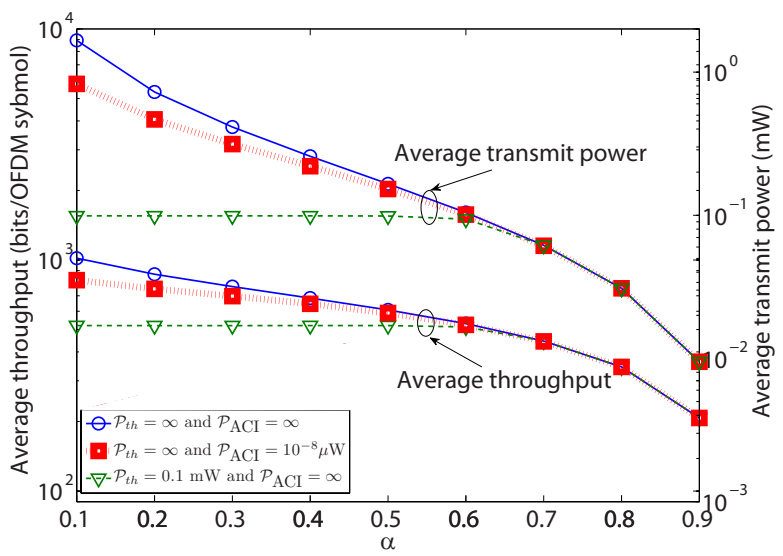

Fig. 2: Effect of the weighting factor $\alpha$ on the OFDM SU performance for different values of $\mathcal{P}_{t h}$ and $\mathcal{P}_{\text {ACI }}$ at $\sigma_{n}^{2}=10^{-3} \mu \mathrm{W}$.

and reference distance $d_{0}=0.5 \mathrm{~km}$. The BER constraint per subcarrier, $\mathrm{BER}_{t h, i_{m}}$, is assumed to be the same for all subcarriers and set to $10^{-4}$. A Rayleigh fading environment is considered with average channel power gain $\mathbb{E}\left\{\left|\mathcal{H}_{i_{m}}\right|^{2}\right\}=$ $\mathbb{E}\left\{\left|\mathcal{H}_{s p}^{(m)}\right|^{2}\right\}=1$. Representative results are presented in this section and were obtained by repeating Monte Carlo trials for $10^{4}$ channel realizations. The PU signal is assumed to be an elliptically filtered white noise process [6], [7], [9], [11], [15] of variance $10^{-3} \mu \mathrm{W}$. Due to space limitations, a certain set of system parameters is chosen to investigate the performance of the proposed algorithm.

Fig. 2 shows the average throughput and average transmit power as a function of the weighting factor $\alpha$ at $\sigma_{n}^{2}=10^{-3}$ $\mu \mathrm{W}$, for different values of $\mathcal{P}_{t h}$ and $\mathcal{P}_{\text {ACI }}$. For $\mathcal{P}_{t h}=\infty$ (inactive $\mathrm{CCI} /$ total transmit power constraint) and $\mathcal{P}_{\mathrm{ACI}}=\infty$ (inactive ACI constraint), one can notice that an increase of the weighting factor $\alpha$ yields a decrease of both the average throughput and average transmit power. This can be explained as follows. By increasing $\alpha$, more weight is given to the transmit power minimization (the minimum transmit power is further reduced), whereas less weight is given to the throughput maximization (the maximum throughput is reduced), according to the problem formulation. Similarly for $\mathcal{P}_{t h}=\infty$ (inactive CCI/total transmit power constraint) and $\mathcal{P}_{\mathrm{ACI}}=10^{-8} \mu \mathrm{W}$ (active ACI constraint), the average throughput and transmit power decrease as $\alpha$ increases. On the other hand, for $\mathcal{P}_{t h}=0.1 \mathrm{~mW}$ (active CCI/total transmit power constraint) and $\mathcal{P}_{\mathrm{ACI}}=\infty$ (inactive $\mathrm{ACI}$ constraint), the same average throughput and power are obtained if the total transmit power is less than $\mathcal{P}_{t h}$, while the average throughput and power saturate if the total transmit power exceeds $\mathcal{P}_{t h}$.

In Fig. 3, the average throughput and average transmit power are plotted as a function of the power threshold $\mathcal{P}_{t h}$, at $\mathcal{P}_{\mathrm{ACI}}=\infty$ (inactive ACI constraint), $\alpha=0.5$, and $\sigma_{n}^{2}=10^{-3}$ $\mu \mathrm{W}$. It can be noticed that the average throughput increases as $\mathcal{P}_{t h}$ increases, and saturates for higher values of $\mathcal{P}_{t h}$; moreover, the average transmit power increases linearly with $\mathcal{P}_{t h}$, while it saturates for higher values of $\mathcal{P}_{t h}$. This can be explained, as for lower values of $\mathcal{P}_{t h}$, the total transmit power is restricted by this threshold value, while increasing this threshold value results in a corresponding increase in both the

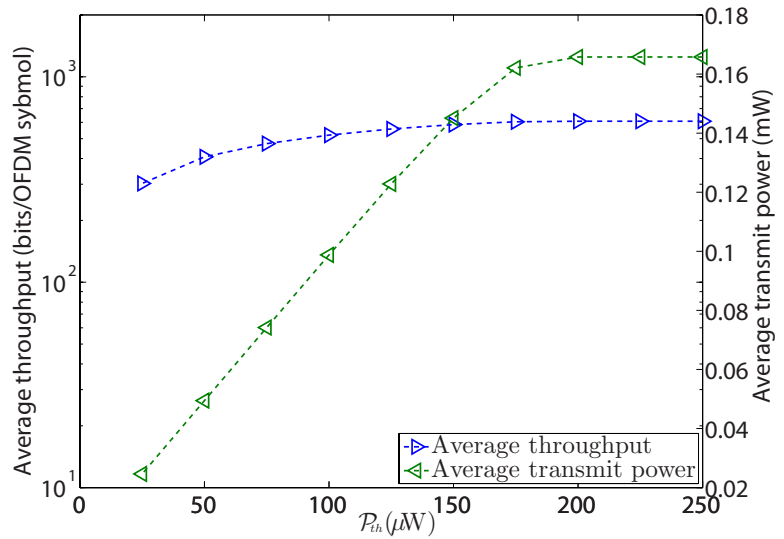

Fig. 3: Effect of $\mathcal{P}_{t h}$ on the OFDM SU performance at $\mathcal{P}_{\mathrm{ACI}}=\infty$, $\alpha=0.5$, and $\sigma_{n}^{2}=10^{-3} \mu \mathrm{W}$.

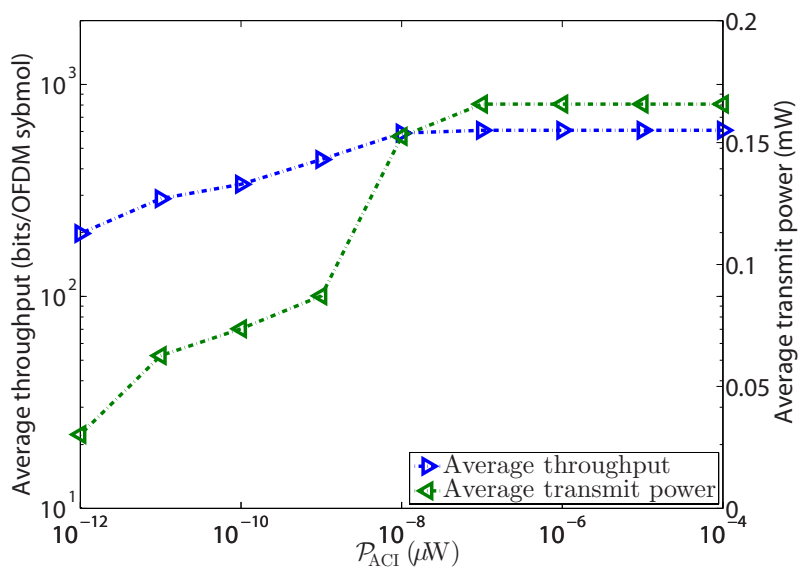

Fig. 4: Effect of $\mathcal{P}_{\mathrm{ACI}}$ on the OFDM SU performance at $\mathcal{P}_{t h}=\infty$, $\alpha=0.5$, and $\sigma_{n}^{2}=10^{-3} \mu \mathrm{W}$.

average throughput and total transmit power. For higher values of $\mathcal{P}_{t h}$, the CCI/total transmit power constraint is inactive. In this case, the proposed algorithm essentially minimizes the transmit power by keeping it constant; consequently, the average throughput remains constant.

Fig. 4 depicts the average throughput and average transmit power as a function of the ACI threshold $\mathcal{P}_{\text {ACI }}$, at $\mathcal{P}_{t h}=\infty$, $\alpha=0.5$ and $\sigma_{n}^{2}=10^{-3} \mu \mathrm{W}$. As can be seen, both the average throughput and average transmit power increase as $\mathcal{P}_{\mathrm{ACI}}$ increases, and saturates for higher values of $\mathcal{P}_{\mathrm{ACI}}$. This can be explained, as for lower values of $\mathcal{P}_{\mathrm{ACI}}$ the ACI constraint is active and, hence, affects the total transmit power. Increasing $\mathcal{P}_{\mathrm{ACI}}$ results in a corresponding increase in both the average throughput and total transmit power. For higher values of $\mathcal{P}_{\mathrm{ACI}}$, the ACI constraint is inactive and the achieved throughput and transmit power saturate.

Fig. 5 compares the objective function achieved with the proposed algorithm and an exhaustive search that finds the discretized global optimal allocation for the problem in (6) for different values of $\mathcal{P}_{t h}$ and $\mathcal{P}_{\text {ACI }}$. Results are presented for a small number of subcarriers $N=8$, such that the exhaustive search is feasible. As one can notice, the proposed algorithm approaches the optimal results of the exhaustive search with significantly reduced computational effort as observed from simulations. 


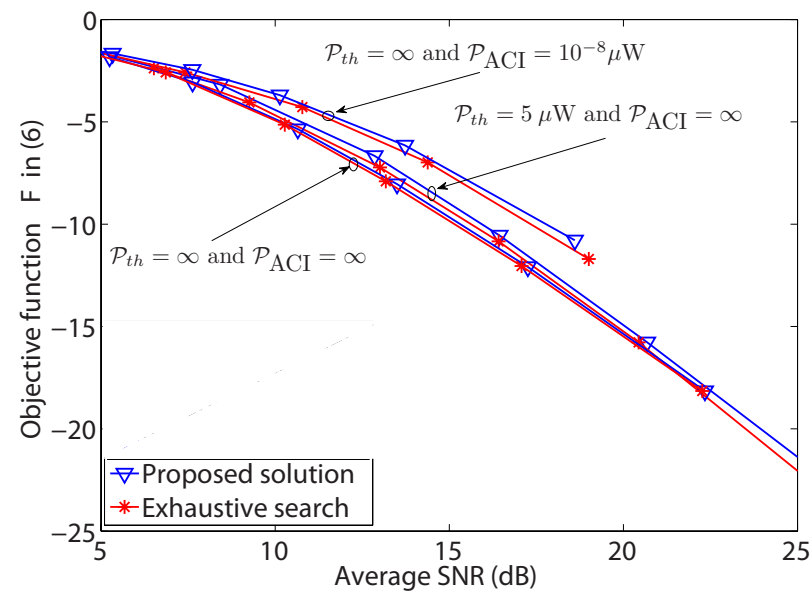

Fig. 5: Objective function for the proposed algorithm and the exhaustive search for different values of $\mathcal{P}_{t h}$ and $\mathcal{P}_{\text {ACI }}$ when $N=8$ and $\alpha=0.5$.

\section{CONCLusions}

In this paper, we proposed a joint bit and power loading algorithm that maximizes the OFDM SU throughput and minimizes its transmit power while guaranteeing a target BER and certain limits on the CCI/total transmit power and ACI interferences to existing PUs. Simulation results show that the proposed algorithm approaches that of an exhaustive search for the discrete optimal allocation.

\section{APPENDIX A}

Proof of the Optimality of $\left(\mathbf{b}_{m}^{*}, \mathbf{p}_{m}^{*}\right)$

The KKT conditions are written as [19]

$$
\begin{aligned}
\frac{\partial \mathcal{F}}{\partial \mathcal{P}_{i_{m}}}+\sum_{\varrho=1}^{N_{m}+2} \lambda_{\varrho} \frac{\partial g_{\varrho}}{\partial \mathcal{P}_{i_{m}}} & =0, \\
\frac{\partial \mathcal{F}}{\partial b_{i_{m}}}+\sum_{\varrho=1}^{N_{m}+2} \lambda_{\varrho} \frac{\partial g_{\varrho}}{\partial b_{i_{m}}} & =0, \\
g_{\varrho} \lambda_{\varrho} & =0, \\
g_{\varrho} & \leq 0, \\
\lambda_{\varrho} & \geq 0,
\end{aligned}
$$

$i_{m}=1, \ldots, N_{m}$ and $\varrho=1, \ldots, N_{m}+2$. One can show that these conditions are satisfied, as sketched in the proof below.

- Proof of (25a) and (25b): one can find that (25a) and (25b) are satisfied from (10a) and (10b), respectively.

- Proof of (25c):

1) In all cases $5-8, \mathcal{Y}_{i_{m}}=0$; hence from (8), $g_{i_{m}}$ is always active, i.e., $g_{i_{m}}=0$.

2) In case 5: $\lambda_{N_{m}+1}=\lambda_{N_{m}+2}=0$; hence, $g_{N_{m}+1} \lambda_{N_{m}+1}=g_{N_{m}+2} \lambda_{N_{m}+2}=0$.

3) In case 6: $\lambda_{N_{m}+2}=0$; hence, $g_{N_{m}+2} \lambda_{N_{m}+2}=0$ and $\mathcal{Y}_{N_{m}+1}=0$, and $g_{N_{m}+1}=0$ from (8).

4) In case 7: $\lambda_{N_{m}+1}=0$; hence, $g_{N_{m}+1} \lambda_{N_{m}+1}=0$ and $\mathcal{Y}_{N_{m}+2}=0$, and $g_{N_{m}+2}=0$ from (8).

5) In case 8: $\mathcal{Y}_{N_{m}+1}=\mathcal{Y}_{N_{m}+2}=0$; hence, $g_{N_{m}+1}=$ $g_{N_{m}+2}=0$ from (8).

Thus, $g_{\varrho} \lambda_{\varrho}=0 \quad \forall \quad \varrho=1, \ldots, N_{m}+2$; hence, (25c) is always satisfied.
- Proof of (25d): adding non-negative slack variables in (8) guarantees that $g_{\varrho} \leq 0$; hence, $(25 \mathrm{~d})$ is always satisfied.

- Proof of (25e): in case 5 and from (10a), one finds

$$
\lambda_{i_{m}}=\alpha\left[0.2 \frac{1.6 \mathcal{C}_{i_{m}}}{2^{b_{i_{m}}}-1} \exp \left(-1.6 \frac{\mathcal{C}_{i_{m}} P_{i_{m}}}{2^{b_{i_{m}}}-1}\right)\right]^{-1},
$$

which is positive for all values of $i_{m}$. Similarly in cases 6,7 and $8, \lambda_{N_{m}+1}$ and $\lambda_{N_{m}+2}$ are found to be positive. Hence, (25e) is always satisfied.

As can be seen, the KKT conditions are satisfied, and, thus, the solution $\left(\mathbf{b}_{m}^{*}, \mathbf{p}_{m}^{*}\right)$ represents a local optimum point.

\section{REFERENCES}

[1] FCC, "Spectrum policy task force," Washington, DC, Rep. ET Docket no. $02-135$, Nov. 2002.

[2] — "Second report and order," Washington, DC, Rep. ET Docket no. 08-260, Nov. 2008.

[3] J. Mitola and G. Maguire, "Cognitive radio: making software radios more personal," IEEE Personal Commun. Mag., vol. 6, no. 4, pp. 1318, Aug. 1999.

[4] E. Hossain and V. Bhargava, Cognitive Wireless Communication Networks. Springer, 2007.

[5] H. Mahmoud, T. Yucek, and H. Arslan, "OFDM for cognitive radio: merits and challenges," IEEE Wireless Commun. Mag., vol. 16, no. 2, pp. 6-15, Apr. 2009.

[6] Z. Hasan, G. Bansal, E. Hossain, and V. Bhargava, "Energy-efficient power allocation in OFDM-based cognitive radio systems: A risk-return model," IEEE Trans. Wireless Commun., vol. 8, no. 12, pp. 6078-6088, Dec. 2009.

[7] C. Zhao and K. Kwak, "Power/bit loading in OFDM-based cognitive networks with comprehensive interference considerations: The singleSU case," IEEE Trans. Veh. Technol., vol. 59, no. 4, pp. 1910-1922, May 2010.

[8] Y. Zhang and C. Leung, "An efficient power-loading scheme for OFDMbased cognitive radio systems," IEEE Trans. Veh. Technol., vol. 59, no. 4, pp. 1858-1864, May 2010.

[9] G. Bansal, M. Hossain, and V. Bhargava, "Optimal and suboptimal power allocation schemes for OFDM-based cognitive radio systems," IEEE Trans. Wireless Commun., vol. 7, no. 11, pp. 4710-4718, Nov. 2008.

[10] X. Kang, Y. Liang, A. Nallanathan, H. Garg, and R. Zhang, "Optimal power allocation for fading channels in cognitive radio networks: ergodic capacity and outage capacity," IEEE Trans. Wireless Commun., vol. 8 , no. 2, pp. 940-950, Feb. 2009.

[11] G. Bansal, M. Hossain, and V. Bhargava, "Adaptive power loading for OFDM-based cognitive radio systems with statistical interference constraint," IEEE Trans. Wireless Commun., no. 99, pp. 1-6, Sep. 2011.

[12] R. Zhang, "Optimal power control over fading cognitive radio channel by exploiting primary user CSI," in Proc. IEEE Global Telecommunications Conference (GLOBECOM), Nov. 2008, pp. 1-5.

[13] E. Bedeer, O. A. Dobre, M. H. Ahmed, and K. Baddour, "Optimal bit and power loading for OFDM systems with average BER and total power constraints," accepted Proc. IEEE Global Communications Conference (GLOBECOM), Dec. 2012.

[14] — "Constrained joint bit and power allocation for multicarrier systems," accepted Proc. IEEE Global Communications Conference (GLOBECOM), Dec. 2012.

[15] T. Weiss, J. Hillenbrand, A. Krohn, and F. Jondral, "Mutual interference in OFDM-based spectrum pooling systems," in Proc. IEEE Vehicular Technology Conference (VTC)-Spring, May 2004, pp. 1873-1877.

[16] T. Rappaport, Wireless Communications: Principles and Practice. Prentice Hall PTR New Jersey, 1996.

[17] T. Willink and P. Wittke, "Optimization and performance evaluation of multicarrier transmission," IEEE Trans. Inf. Theory, vol. 43, no. 2, pp. 426-440, Mar. 1997.

[18] S. Chung and A. Goldsmith, "Degrees of freedom in adaptive modulation: a unified view," IEEE Trans. Commun., vol. 49, no. 9, pp. 15611571, Sep. 2001.

[19] S. Boyd and L. Vandenberghe, Convex Optimization. Cambridge University Press, 2004.

[20] R. Burden and J. Faires, Numerical Analysis. Cengage Learning, 2010. 